\title{
Erratum to: Macro-foundations for Fiscal Analysis
}

\section{Erratum to: \\ Chapter 2 in: A. J. Makin, The Limits of Fiscal Policy, https://doi.org/10.1007/978-3-319-90158-9_2}

The previous version of this book incorrectly displayed figure 2.2 in Chapter 2 which has been corrected in this version. The erratum chapter and the book have been updated with the change.

The updated online version of this chapter can be found at https://doi.org/10.1007/978-3-319-90158-9_2

(C) The Author(s) 2018 